\title{
CARACTERÍSTICAS CLÍNICO EPIDEMIOLÓGICAS DE LA ENFERMEDAD DE PARKINSON EN UN HOSPITAL NACIONAL DE LA SIERRA PERUANA
}

\author{
CLINICAL EPIDEMIOLOGICAL CHARACTERISTICS OF PARKINSON'S DISEASE IN A NATIONAL \\ HOSPITAL OF THE PERUVIAN HIGHLANDS
}

Iván R. Condor ${ }^{1, a}$, Joel I. Atencio-Paulino ${ }^{1, b}$, Coco R. Contreras-Cordova ${ }^{1, c}$

\begin{abstract}
RESUMEN
Objetivo: Determinar las principales características epidemiológicas y clínicas de la enfermedad de Parkinson en el Hospital Nacional "Ramiro Prialé Priale" de Huancayo. Métodos: Estudio observacional, descriptivo tipo retrospectivo de una serie de casos. Se estudió 84 pacientes entre los años 2015 y 2017, atendidos en la consulta externa u hospitalización de Neurología y Medicina interna, para ello se revisó sus historias clínicas, el muestreo fue no probabilístico de tipo censal seleccionados por juicio del investigador. Resultados: El promedio de edad fue 72,93 años, predominó el género masculino (58,3\%), el grado de instrucción universitario $(50 \%)$ y la procedencia de Huancayo $(77,4 \%)$. La hipertensión arterial descompensada fue el motivo de hospitalización $(19,1 \%)$, el tiempo de enfermedad tuvo un rango de 1-7 años $(57,1 \%)$ y el temblor de reposo y bradicinesia fue el síntoma motor más frecuente $(23,8 \%)$. En los síntomas no motores, 38,1\% tuvieron alteraciones del sueño, 21,4\% antecedentes familiares de enfermedad de Parkinson y las comorbilidades más asociadas fueron: depresión $(48,8 \%)$ e insomnio $(41,7 \%)$. Conclusión: La enfermedad de Parkinson es una patología frecuente en adultos mayores y en el sexo masculino; el síntoma motor que predomina es el temblor de reposo y bradicinesia, y las comorbilidades neuropsiquiátricas asociadas son la depresión e insomnio.
\end{abstract}

Palabras clave: Enfermedad de Parkinson; Hipocinesia; Trastornos del inicio y del mantenimiento del sueño. (fuente: DeCS BIREME)

\begin{abstract}
Objective: To determine the main epidemiological and clinical characteristics of Parkinson's disease at the National Hospital "Ramiro Prialé Priale" in Huancayo. Methods: Observational, descriptive retrospective study of a serie of cases. 84 patients were studied between 2015 and 2017, treated in the outpatient clinic or hospitalization of Neurology and Internal Medicine, for which their medical records were reviewed, the sampling was non-probabilistic of the census type selected by the investigator's judgment. Results: The average age was 72.93 years, the male gender prevailed (58.3\%), the degree of university education (50\%) and the origin of Huancayo (77.4\%). Decompensated arterial hypertension was the reason for hospitalization (19.1\%), disease time ranged between 1-7 years (57.1\%) and tremor at rest and bradykinesia was the most frequent motor symptom (23.8\%) In non-motor symptoms, 38.1\% had sleep disorders, $21.4 \%$ family history of Parkinson's disease and the most associated comorbidities were: depression (48.8\%) and insomnia (41.7\%). Conclusion: Parkinson's disease is a frequent pathology in older adults and males; The predominant motor symptom is resting tremor and bradykinesia, and the associated neuropsychiatric comorbidities are depression and insomnia.
\end{abstract}

Key words: Parkinson's disease; Hypokinesia; Disorders of onset and maintenance of sleep. (source: MeSH NLM)

\footnotetext{
'Facultad de Medicina Humana, Universidad Nacional del Centro del Perú, Huancayo - Perú.

a Médico cirujano.

${ }^{\mathrm{b}}$ Estudiante de medicina.

${ }^{c}$ Médico Geriatra.

Citar como: Iván R. Condor, Joel I. Atencio-Paulino, Coco R. Contreras-Cordova. Características clínico epidemiológicas de la enfermedad de parkinson en un hospital nacional de la sierra peruana. Rev. Fac. Med. Hum. Octubre 2019; 19(4):14-21. DOI 10.25176/RFMH.v19i4.2342
} 


\section{INTRODUCCIÓN}

La enfermedad de Parkinson según la OMS, en el año 2016 su afección era una persona por cada 100 personas mayores de 60 años, siendo en cifras absolutas 6,3 millones de personas, y para el año 2030 serán aproximadamente 12 millones de personas en todo el mundo con dicha patología'. En el 2008, la OPS identificó que los pacientes con epilepsia, enfermedad de Parkinson y Alzheimer en conjunto significaban más del $6 \%$ de la carga global para las instituciones médicas ${ }^{2}$. La enfermedad de Parkinson en los Estados Unidos afecta aproximadamente a más de un millón de personas, siendo en su gran mayoría personas mayores de 50 años $^{3}$.

En el Perú, no se tienen cifras oficiales o exactas, el Instituto Nacional de Ciencias Neurológicas (INCN) estimó en el año 2014 que el 1\% de la población en general tenía enfermedad de Parkinson, es decir un

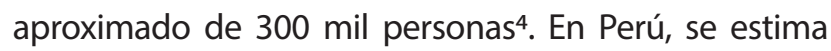
que para el año 2030 serán 2015065 las personas con enfermedad de Parkinson y la tasa de mortalidad de 25,45 por cada 100 mil personas 5 . Cada año se notifican entre 2000 y 3000 casos nuevos en nuestro país, y en la ciudad de Lima serían aproximadamente $10 \mathrm{mil}$ personas con esta enfermedad6.

El seguro social de salud EsSalud, trata de brindar una atención más integral, desde terapias de rehabilitación hasta medicamentos adicionales en caso de presentar comorbilidades y cirugía por estimulación en casos severos (aplicada desde el año 2007)7.

Se realizó un estudio sobre la prevalencia de esta enfermedad siendo la región Junín el segundo lugar respecto al nacimiento y procedencia después de Lima metropolitana, además se determinó su relación con tóxicos, en especial con el uso de algunos pesticidas ${ }^{8}$. El objetivo de la presente investigación es determinar las principales características (clínicas, epidemiológicas, tratamiento y comorbilidades) de la enfermedad de Parkinson.

\section{MÉTODOS}

\section{Tipo y diseño}

Estudio observacional tipo retrospectivo donde se analizó una serie de casos clínicos, el lugar de ejecución fue el Hospital Ramiro Prialé Prialé de Huancayo.

\section{Población y muestra}

Pacientes que cumplían con los criterios de enfermedad de Parkinson, atendidos en los consultorios externos y/o en la sala de hospitalización de los servicios de neurología y/o medicina interna, entre los meses de julio del 2015 a julio del 2017, en el Hospital Ramiro Prialé Prialé.

Se decidió estudiar toda la población disponible, en este caso de tipo censal, que fue aproximadamente 120 pacientes que luego de la selección con los criterios planteados por los investigadores quedaron 84 . Además, el muestreo fue no probabilístico siendo por criterio o juicio de los investigadores ${ }^{9,10}$; se incluyeron las historias clínicas de pacientes con diagnóstico de enfermedad de Parkinson que fueron hospitalizados en el servicio de medicina interna $y / o$ atendidos en los consultorios externos de neurología del Hospital Nacional "Ramiro Prialé Prialé" de Huancayo durante los años 2015 y 2017, descartando las historias clínicas tengan los registros incompletos.

\section{Métodos de análisis y recolección de datos}

Se elaboró una ficha para cada paciente con enfermedad de Parkinson, que contiene los parámetros: epidemiológicos, clínicos y de comorbilidades, validada por juicio de expertos, siendo esta validación por criterio o contenido por docentes de la Facultad de Medicina Humana-Universidad Nacional del Centro del Perú (UNCP), así mismo se realizó una prueba piloto para ver la confiablidad de la ficha de recolección de datos resultando una buena confiablidad con un alfa de Cronbach 0,81.

\section{Análisis estadístico}

En primer lugar, se utilizó la estadística descriptiva para los datos cuantitativos, se usaron las medidas de tendencia central y de dispersión; para variables cualitativas o categóricas frecuencias y porcentajes, así mismo el uso de graficas como el diagrama barras. Se utilizó para el análisis programas estadísticos como el SPSS 24, Epi Info 4, Med Clac y Excel 2016. La recolección y análisis estuvo a cargo de los autores que participaron en la presente investigación.

\section{Consideraciones éticas}

El siguiente trabajo de investigación se realizó con la debida autorización de la institución con la seguridad que los datos obtenidos sean utilizados únicamente para fines científicos, conservando el anonimato de todos los pacientes, al igual con los lineamientos de ética para la investigación que promueve el Hospital Nacional "Ramiro Prialé Prialé" de Huancayo.

\section{RESULTADOS}

Se revisó 84 historias clínicas que cumplían con los criterios de selección de los investigadores, en la Tabla 
1, se puede ver las características epidemiológicas, el promedio de edad fue 72,93 años con un IC al 95\%: (54,71-91,15), con una desviación estándar 9,298 siendo el minino 50 y máximo 90 años; respecto al género fue más frecuente el masculino (58,3\%), también predominó el estado civil casado (69\%), el grado de instrucción superior universitario (50\%) y Huancayo como lugar de procedencia $(77,4 \%)$.

En las características clínicas el motivo de hospitalización fue en primer lugar por hipertensión arterial descompensada (19,1\%) seguido de colecistitis crónica $(11,9 \%)$ (Tabla 2 ), el tiempo de enfermedad fue entre 1 año y 7 años; en los síntomas motores se describió que el más frecuente fue el temblor de reposo y bradicinesia $(23,8 \%)$, en cuanto a los miembros afectados el (33,3\%) tenían afección de todas las extremidades (Tabla 2). En los síntomas no motores el más frecuente fue alteraciones del sueño $(38,1 \%)$; en los síntomas autonómicos la presencia de sialorrea (19\%), en los síntomas urinarios la urgencia de orinar (38,1\%); finalmente en los síntomas sensoriales el $72,6 \%$ refieren dolor y $10,7 \%$ ageusia. El tratamiento pilar para la enfermedad de Parkinson es el levodopa/carbidopa $(51,2 \%)$ y en los tratamientos adicionales el uso de anticolinérgicos $(60,7 \%)$ (Tabla 3). En los factores intrínsecos el 21,4\% refieren tener antecedentes familiares de enfermedad de Parkinson. Por último, la comorbilidad de mayor frecuencia fue la depresión (48,8\%) (Tabla 3 y Figura 1).

Tabla 1. Características epidemiológicas de los pacientes con enfermedad de Parkinson del Hospital Ramiro Prialé Prialé de Huancayo.

\begin{tabular}{|c|c|c|}
\hline & Media & IC $95 \%$ \\
\hline $\operatorname{Edad}(n=84)$ & $72,93 \pm 9,30$ & $54,71-91,15$ \\
\hline Género $(n=84)$ & (n) & (\%) \\
\hline Masculino & 49 & 58,3 \\
\hline Femenino & 35 & 41,7 \\
\hline Estado civil (n=84) & (n) & (\%) \\
\hline Casado & 58 & 69,0 \\
\hline Soltero & 19 & 22,6 \\
\hline Viudo & 6 & 7,1 \\
\hline Divorciado & 1 & 1,2 \\
\hline Grado de instrucción ( $n=84)$ & (n) & (\%) \\
\hline Sin Grado de instrucción & 3 & 3,6 \\
\hline Primaria & 5 & 6,0 \\
\hline Secundaria & 23 & 27,4 \\
\hline Superior no universitario & 11 & 13,1 \\
\hline Superior universitario & 42 & 50,0 \\
\hline Procedencia $(n=84)$ & (n) & (\%) \\
\hline Huancayo & 65 & 77,4 \\
\hline Jauja & 5 & 6,0 \\
\hline Concepción & 3 & 3,6 \\
\hline Tarma & 3 & 3,6 \\
\hline Yauli & 1 & 1,2 \\
\hline Junín & 1 & 1,2 \\
\hline Otros & 6 & 7,1 \\
\hline
\end{tabular}


Tabla 2. Características clínicas de los pacientes con enfermedad de Parkinson del Hospital Ramiro Prialé Prialé de Huancayo.

Motivo de hospitalización( $(n=41)$

Hipertensión arterial descompensada

Colecistitis crónica

Traumatismo clavícula

Traumatismo olecranon

Hernia ventral

Traumatismo de cadera

Traumatismo de pelvis

Venas varicosas

Tiempo de enfermedad $(n=84)$

Entre 1 año y 7 años

Entre 8 años y 14 años

Mayor de 14 años

Menor de 1 año

Síntomas motores $(n=78)$

Temblor de reposo y bradicinesia

Temblor de reposo y rigidez

Temblor de reposo, bradicinesia e inexpresividad facial

Temblor de reposo, bradicinesia e inestabilidad postural

Temblor de reposo y movimiento

Temblor de reposo

Bradicinesia

Otros

Miembros afectados(n=84)

\section{(n)}

(\%)

19,1

10

11,9

\section{4}

3

3,6

2

2,4

$2 \quad 2,4$

2

2,4

2

2,4

(n)

(\%)

48

57,1

24

28,6

9

10,7

3

3,6

(n)

(\%)

20

23,8

15

17,9

12

14,3

8

9,5

7

8,3

5

6,0

5

6,0

6

7,2

Todos los miembros 33,3

Extremidad superior e inferior derecha

Extremidad superior e inferior izquierda

Ambas extremidades superiores

Extremidad superior izquierda

\section{6} 7,1

Extremidad superior derecha

Extremidad superior derecha e inferior izquierda

Otros

3 
Tabla 3. Síntomas no motores y comorbilidades de los pacientes con enfermedad de Parkinson del Hospital Ramiro Prialé Prialé de Huancayo.

Síntomas no motores $(n=56)$

Alteraciones del sueño

Alteraciones del pensamiento

Alteraciones del pensamiento y del sueño

Alteraciones del lenguaje

Alteraciones del pensamiento, sueño y del lenguaje

Alteraciones de la memoria

Síntomas autonómicos $(\mathrm{n}=27)$

Sialorrea

Alteraciones gastrointestinales

Hipotensión ortostática

Síntomas urinarios $(\mathrm{n}=58)$

Urgencia de orinar

Nicturia

Incremento de frecuencia

Síntomas sensoriales $(n=84)$

Dolor

Ageusia

Dolor y acatisia

Dolor y parestesias

Parestesias

Tratamiento $(\mathrm{n}=80)$

Levodopa- carbidopa

Levodopa

Tratamientos adicionales $(\mathrm{n}=81)$

Anticolinérgicos

Anticolinérgicos y selegilina

Selegilina y/u Otro antidepresivo

Anticolinérgicos y agonistas dopaminérgicos

Agonistas dopaminérgicos y selegilina 


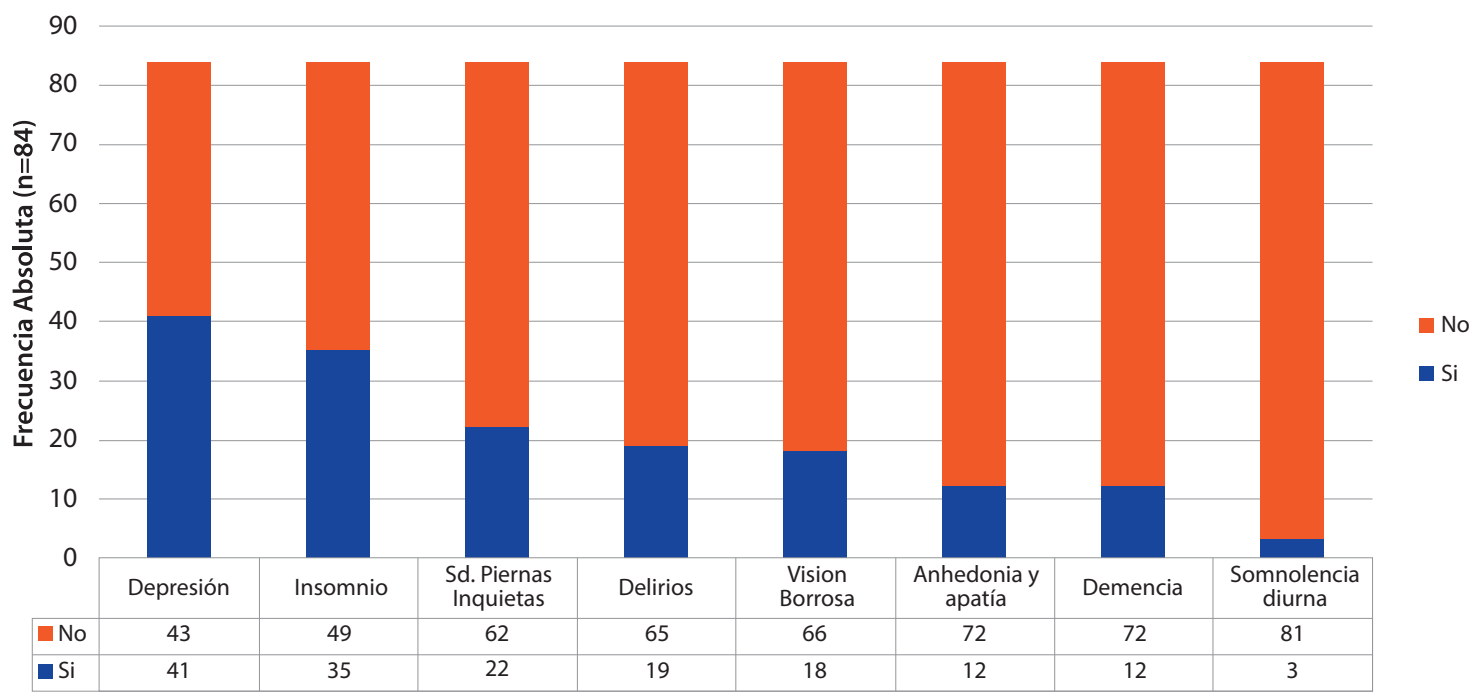

Figura 1. Comorbilidades presentes en los pacientes con enfermedad de Parkinson del Hospital Ramiro Prialé Prialé de Huancayo (Frecuencias absolutas).

\section{DISCUSIÓN}

En nuestro estudio el promedio de edad fue 72,93 años, el género más frecuente fue el masculino $(58,3 \%)$ y los grados de instrucción más prevalentes fueron superior universitario (50\%), y secundaria (27,4\%). Resultados similares se encontró en Ecuador, el promedio de edad fue 62,3 años y predominó en el género masculino $(53,8 \%)^{11}$. En Chile, también predominó en el género masculino $(58,73 \%)$ y la edad promedio fue 71,77 años, pero encontramos diferencia respecto al grado de instrucción, en donde los pacientes sin estudio superiores representaron el $58,73 \%^{12}$. En otro estudio realizado en el Perú, si hubo diferencias estadísticas importantes en cuanto a las frecuencias encontradas con respecto al género masculino $(28,6 \%)$ y la edad promedio $(55,95 \text { años })^{8}$.

En las características clínicas identificamos que respecto al tiempo de enfermedad, más de la mitad, tuvo entre 1 año y 7 años; en los síntomas motores se describió que el más frecuente fue el temblor de reposo y bradicinesia. Según Martín ${ }^{13}$, determinó que el tiempo de enfermedad de los pacientes con enfermedad Parkinson era 7,36 años. El Instituto Nacional de Ciencias Neurologías (INCN) estableció que el $42,86 \%$ tenían un tiempo de enfermedad entre 5-9 años y que los síntomas motores más prevalentes en orden de mayor a menor frecuencia fueron: bradicinesia $(87,3 \%)$, rigidez $(84,16 \%)$, temblor de reposo $(79,37 \%)$ y alteración postural $(74,6 \%)^{14}$. En la misma línea Cuba J, et al., en la caracterización de los síntomas motores la afección en los pacientes que tenían una edad menor de 40 años tuvo una alteración unilateral, siendo la afección del miembro superior $(65,30 \%)$, y del miembro inferior $(34,7 \%)^{8}$.

Núñez-Peralta ${ }^{15}$, reporta que los síntomas motores más frecuentes son la rigidez $(69,4 \%)$, el temblor de reposo $(64,2 \%)$ y la bradicinesia $(42,5 \%)$ y los síntomas no motores las alteraciones del sueño $(38,1 \%)$ y la urgencia de orinar (38,1\%). Además, predomina el uso de anticolinérgicos (60,7\%); el 21,4\% refiere tener antecedentes familiares de enfermedad de Parkinson y las comorbilidades más frecuentes fueron depresión $(48,8 \%)$ e insomnio $(41,7 \%)$.

Según Navarro ${ }^{16}$, en el tratamiento farmacológico de dichos pacientes la combinación de dopamina y otras drogas antiparkinsonianos estuvieron presentes en el 52,5\%. En cuanto a los antecedentes de enfermedad de Parkinson si hubo diferencias con el estudio realizado en Chile, donde posiblemente al ser una institución especializada ellos determinaron que el $100 \%$ de sus pacientes si tenían antecedentes familiares $^{12}$, en nuestro estudio sólo la quinta parte tuvo antecedentes $(21,4 \%)$, es importante el estudio de la "Dinámica Mitocondrial" en ciertos pacientes, aunque en general se sabe que en la gran mayoría de los pacientes con enfermedad de Parkinson existe un componente multifactorial ${ }^{17,18}$.

Con respecto a las comorbilidades neuropsiquiátricas asociadas a la enfermedad de Parkinson, Martin ${ }^{13}$ identificó en su estudio la presencia de demencia $(12 \%)$, alteraciones del sueño $(81,36 \%)$ y depresión $(37,1 \%)$. En nuestro país, se identificó que dentro de 
las complicaciones que desarrollan estos pacientes es muy frecuente la depresión $(42,85 \%)^{8,19,20}$; NúñezPeralta $^{15}$ determinó, en los síntomas no motores, que la depresión estuvo presente en el $26,9 \%$ de los pacientes.

La limitación de nuestro trabajo de investigación es que al ser un estudio observacional, retrospectivo y de serie de casos, sólo se describen las características de este grupo de pacientes con enfermedad de Parkinson, lo que probablemente no genere evidencia que permita alterar la práctica clínica, pero si su vigilancia epidemiológica, conocer el cuadro clínico y poder evaluar la mejor metodología para investigaciones posteriores.

\section{CONCLUSIÓN}

Se determinó que la enfermedad de Parkinson es una patología que principalmente afecta a personas mayores del sexo masculino, el síntoma motor más característico es el temblor de reposo y bradicinesia y los síntomas no motores las alteraciones del sueño como el insomnio y la depresión.
Contribuciones de autoría: Los autores participaron en la génesis de la idea, diseño de proyecto, recolección e interpretación de datos, análisis de resultados y preparación del manuscrito del presente trabajo de investigación.

\section{Financiamiento: Autofinanciado.}

Conflicto de interés: Los autores declaran no tener conflicto de interés en la publicación de este artículo.

Recibido: 20 de marzo 2019

Aprobado: 29 de agosto 2019

\section{Correspondencia: Joel Atencio}

Dirección: Av. Manantial 4817, Sapallanga- Huancayo; código postal: 12000 Teléfono: 927999363

Correo:yoel_005@yahoo.com

\section{REFERENCIAS BIBLIOGRÁFICAS}

1. Parkinson y yo, Organización Mundial de la Salud (OMS) [Internet]. Buenos Aires, Argentina: Parkinson y yo, Acerca del Parkinson [Actualizado 2016 Dic; citado el 1 Feb. de 2017]. Disponible en:

http://terapiaparkinson.com/testimonial-view/organizacion-mundialde-la-salud-oms/

2. Organización Panamericana de la Salud, trastornos neurológicos: un serio desafío para la salud pública en las Américas y en todo el mundo [Internet]. Washington, Estados Unidos: Organización Panamericana de la Salud, Enfermedades no transmisibles y salud mental [Actualizado 2008; citado el 1 Feb. de 2017]. Disponible en:

http://www.paho.org/hq/index.php?option=com_content\&vie $\mathrm{w}=$ article\&id $=240 \% 3 \mathrm{~A} 2008$-trastornos-neurologicos-un-seriodesafio-salud-publica-americas-todo-mundo\&catid=916\%3Ariskfactors\&ltemid=40595\&lang=es

3. American Parkinson Disease Association, Understanding the Basic of parkinson's Disease [Internet]. New York, Estados Unidos: American Parkinson Disease Association, What is Parkinson's Disease? [Actualizado 2017; citado el 1 Feb. de 2017]. Disponible en:

https://www.apdaparkinson.org/what-is-parkinsons/

4. Diario Médico, Parkinson avanzados es controlable en el Perú [Internet]. Lima, Perú: Diario Médico, Estimulación cerebral profunda Parkinson [Actualizado 2014 Jun; citado el 1 Feb. de 2017]. Disponible en: http://www.diariomedico.pe/?p=6810

5. Organización Mundial de la Salud. Trastornos Neurológicos, desafíos para la salud pública. Estados Unidos: Organización Mundial de la Salud; 2006. 211p. Disponible en: http://www1.paho.org/hq/ dmdocuments/2008/Trastornos_Neurologicos.pdf

6. Perú 21, Solo en Lima habría 10,000 personas con Parkinson [Internet]. Lima, Perú: Perú 21, Redacción Peru21 [Actualizado 2017 Abr; citado el 1 Feb. de 2017]. Disponible en: https://peru21.pe/lima/lima-habria-10 000-personas-parkinson-72412
7. EsSalud, EsSalud ofrece tratamiento integral para enfermedad de Parkinson a sus asegurados [Internet]. Lima, Perú: EsSalud, Noticias [Actualizado 2014 Abr; citado el 1 Feb. de 2017]. Disponible en: http:// www.essalud.gob.pe/essalud-ofrece-tratamiento-integral-paraenfermedad-de-parkinson-a-sus-asegurados/

8. Cuba J, Cosentino C, Díaz A, Torres L, Martinot C. Algunos aspectos Clínico - Epidemiológicos de los síndromes parkinsonianos en un servicio de Neurología en 25 años. Revista Peruana de Neurología. 1995; 1(2):70-73. Disponible en: http://sisbib.unmsm.edu.pe/BVRevistas/ neurologia/v01_n2/algunos.htm

9. Supo J. Taxonomía de la investigación: El arte de clasificar aplicado a la investigación científica. 2da ed. Arequipa: Bioestadístico; 2015.

10. Supo J. Cómo se elige una prueba estadística: 6 criterios para elegir un procedimiento estadístico. 1ra ed. Arequipa: Bioestadístico; 2013.

11. Andino-Núñez A. Enfermedades neurodegenerativas en el Hospital de Especialidades Eugenio Espejo, período 2000-2012, Quito-Ecuador. [Tesis]. Quito: Colegio de Ciencias de la Salud, Universidad San Francisco de Quito; 2013. Disponible en: http://repositorio.usfq.edu.ec/ handle/23000/2235

12. Solís M, Araneda J. Enfermedad de Parkinson y factores ambientales. Un estudio caso-control. Revista Chilena de Neuropsiquiatría. 2017; 55(4):1-8. Disponible en: http://dx.doi.org/10.4067/s071792272017000400239

13. Martín Balbuena S. Detección e interrelación de los síntomas no motores más comunes en la enfermedad de Parkinson. Estudio multicéntrico observacional descriptivo de serie de casos en la región de Murcia. [Tesis Doctoral]. Murcia: Departamento de Medicina Interna, Universidad de Murcia; 2015. Disponible en: https://dialnet.unirioja.es/ servlet/tesis?codigo $=155250$

14. Rodríguez O, Pezo, Torres L. Asociación entre Características clínicas de pacientes con enfermedad de Parkinson y su desempeño en la marcha en tándem. CIMEL. 2017; 22(2):9-13. Disponible en: https://doi. org/10.23961/cimel.v22i2.766 
15. Núñez-Peralta C. Perfil clínico de la enfermedad de Parkinson en el servicio de neurología del hospital nacional Alberto sabogal Sologuren, periodo enero - diciembre del año 2013. [Tesis]. Lima: Facultad de Medicina Humana, Universidad Ricardo Palma; 2014. Disponible en: http://repositorio.urp.edu.pe/handle/urp/300

16. Navarro-Peternella F. Calidad de vida de las personas con enfermedad de Parkinson y su relación con la evolución en el tiempo y la gravedad de la enfermedad. Revista Latino-Am. Enfermagem. 2012; 20(2): 1-8. Disponible en: http://dx.doi.org/10.1590/S0104-11692012000200023

17. Benito-León J. Epidemiologia de la enfermedad de Parkinson en España. Revista de Neurología. 2018; 66(4):125-34. Disponible en: https://doi.org/10.33588/rn.6604.2017440
18. Archer S. Mitochondrial Dynamics - Mitochondrial Fission and Fusion in Human Diseases. The New England Journal of Medicine. 2013 Dic; 369(23):2236-2251. Disponible en: https://doi.org/10.1056/ NEJMra1215233

19. Torres L. Epidemiologia de la enfermedad de Parkinson. Revista de Neuropsiquiatría. 1998; 61(1):8-13. Disponible en: https://doi. org/10.20453/rnp.v61i5.1442

20. Torres L, Mori N, Cuentas M, Domínguez J, Mendoza M, Montoya $J$, et al. Prevalencia de la enfermedad de Parkinson, Un estudio puerta a Puerta en Cinco distritos de Ulcumayo - Junín, Perú. Revista Diagnostico. 2008; 47(4):150-56.

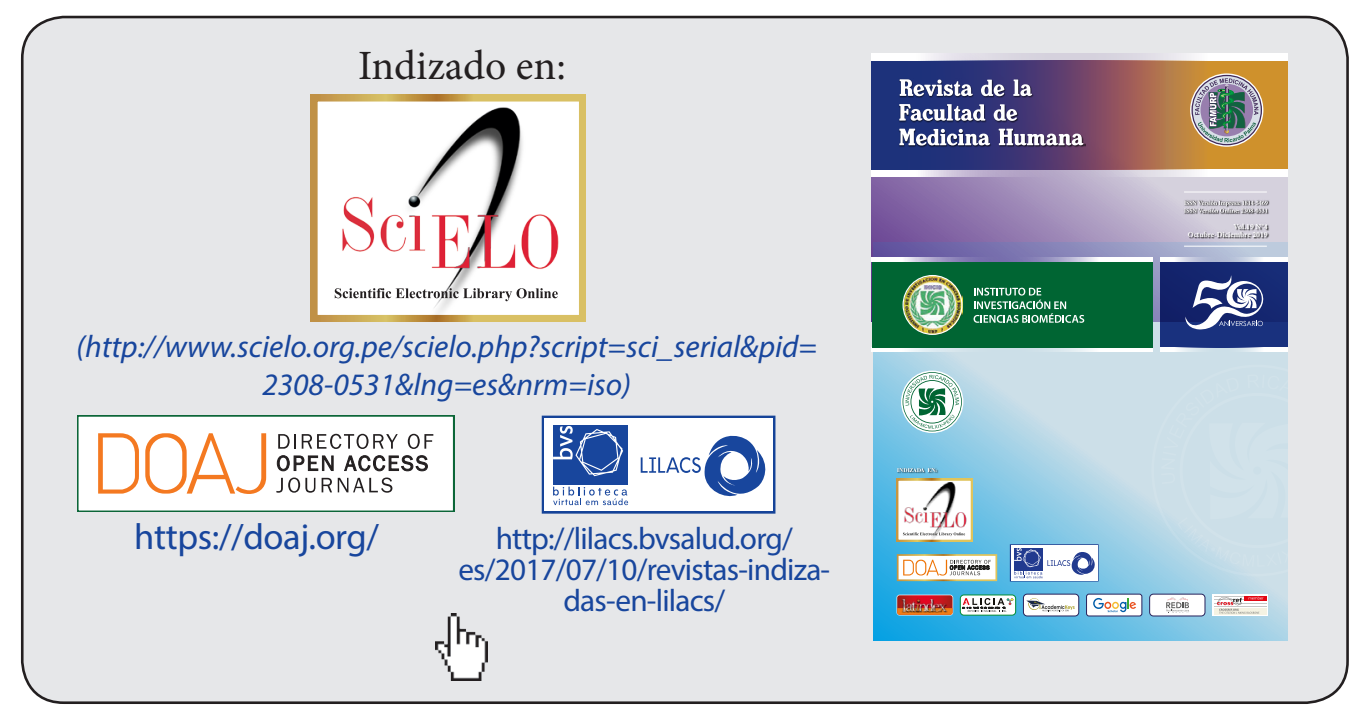

